\title{
EFFECT OF THYMOQUINONE ON WOUND HEALING IN ALLOXAN-INDUCED DIABETIC RATS
}

\author{
AHMADI YUSMIN, NORHAYATI AHMAD*
}

Environmental and Life Sciences Programme, Faculty of Science, Universiti Brunei Darussalam, Jalan Tungku Link, Gadong BE1410, Brunei Darussalam. Email: norhayati.ahmad@ubd.edu.bn

Received: 06 April 2017, Revised and Accepted: 30 May 2017

ABSTRACT

Objective: Nigella sativa and its active constituent thymoquinone (TQ) have been extensively documented for its pharmacological values, but its application in wound healing in particular in a diabetic wound healing model is less documented.

Methods: In our study, alloxan-induced diabetic rats were used as a chronic delayed wound model and topical administration of TQ $10 \%$ w/v were used to assess the role and function of TQ in wound healing through wound contraction and histological analysis.

Results: Although statistically insignificant, we found out that TQ accelerated wound healing in post-wounding day 3 (inflammatory phase), whereas aggressively decelerating wound healing in post-wounding day 7 (proliferation phase). In addition, our histological analyses of wound granulation tissues at post-wounding day 14 substantiate our claim by showing that TQ treatment had delayed wound healing progression of the diabetic rats.

Conclusions: Our study shows that TQ accelerates wound healing during the inflammatory phase; however, decelerate rapidly during the proliferation phase. We speculate the acceleration of wound healing during the inflammatory phase was due to its well-documented antioxidant, anti-inflammatory, and antimicrobial properties while its deceleration of wound healing during the proliferation phase was due to its well-documented antiangiogenic effect.

Keywords: Wound healing, Thymoquinone, Nigella sativa, Diabetes mellitus.

(C) 2017 The Authors. Published by Innovare Academic Sciences Pvt Ltd. This is an open access article under the CC BY license (http://creativecommons. org/licenses/by/4. 0/) DOI: http://dx.doi.org/10.22159/ajpcr.2017.v10i9.18951

\section{INTRODUCTION}

Naturally, cutaneous wound heals spontaneously and does not necessitate any medical aid [1]. It is a continual, overlapping but independent event occurring in four described phases mainly hemostasis, inflammation, proliferation (early granulation), and remodeling (late granulation) with their own standard time frame.

Chronic non-healing ulceration is commonly associated with diabetes mellitus affecting approximately $2-4 \%$ of patients [2-4]. Ulceration is as a result of collective tissue breakdown manifested by impaired healing [4,5]. This is primarily associated with a series of macrovascular and microvascular alteration that leads to many systemic complications $[4,6]$. These systemic complications will result in delayed wound healing that may eventually lead to limb amputation $[4,7]$. Nontraumatic lower limb amputation is one of the worst complications of diabetes. Treatment is naturally difficult due to predominance of polymicrobial with multidrug resistance and capability of biofilm formation [8].

Most modern medicines today are derived from the study of medicinal plants. Plants have been phytochemically screened and tested for the past 210 years [9]. It is appealing to consider effective phytochemicals such as to treat against bacterial infection and the same time, improve inflammatory and angiogenic functions in wound healing.

Thymoquinone (TQ) (2-methyl-5-isopropyl-1,4-benzoquinone) was first phytochemically screened and isolated by El-Dakhakhny [10]. As of yet, in vivo model has yet been employed to evaluate wound healing ability of Nigella sativa and its active constituent TQ. The nearest preclinical trial of such was using a scratch assay of fibroblast migration using $N$. sativa seed extract described recently by Ab Rahman et al. [11] TQ strong antioxidant-associated capacities and strong antimicrobial property, all suggested that TQ is a desirable wound healing alternative [12]. Due to TQ high pharmacological value and the severity of chronic non-healing complication, it is imperative to explore the medicinal activity of TQ in wound healing therapy. Hence, our present study was conducted to investigate to determine the effect of $\mathrm{TQ}$ in the wound healing progression of alloxan-induced diabetic rats.

\section{METHODS}

Animal

Male Wistar rats aged 10-12 weeks were used in all experiments. Approval for work and procedures with animals has been granted by the UBD University Research Ethics Committee. Rats were kept under optimal controlled humidity on a 12 hrs light/dark circle. Feeding pellets and sufficient access to clean water were provided.

\section{Diabetes induction}

Animals were fasted overnight with access to water ad libitum. Alloxan monohydrate (Sigma Chemicals, USA) was freshly dissolved in $0.9 \%$ saline solution $(\mathrm{w} / \mathrm{v})$. Animals were administered with a single dose of alloxan administration at $120 \mathrm{mg} / \mathrm{kg}$ through the intraperitoneal route.

Blood glucose levels were measured at 36 hrs and $72 \mathrm{hrs}$ after alloxan administration to confirm hyperglycemia and before the experimental conduct. During the course of the experiment, blood glucose levels and body weight of rats were recorded at day 0 , day 7 , and day 14 to ensure successful diabetes induction. Blood glucose parameter was as previously described [13], rats with blood glucose readings of $\geq 300 \mathrm{mg} / \mathrm{dL}$ were used for the study.

\section{Wound excision}

Rats were anesthetized using ether fume inhalation method as previously described [14]. Excisional wounds were made as previously described with slight modifications [15]. Dorsal skin was shaved using electric razor and cleaned using repeated application of ethanol. An excision full thickness of approximately $5 \mathrm{~mm}$ was made to the size of approximately $225 \mathrm{~mm}^{2}(15 \times 15 \mathrm{~mm})$ using sterilized 
surgical equipment. Wounds were cleaned with repeated flushing of $0.9 \% \mathrm{w} / \mathrm{v}$ saline solution and dried with cleaned gauze. The first topical application was then administered to the wound with their own assigned treatments. Wounds were left exposed uncovered and monitored daily

\section{Groupings and treatments}

Diabetic rats were grouped into two groups at 5 animals per group. Control vehicle only group was administered topically with white petroleum jelly (Vaseline ${ }^{\circledR}$ Jelly) while treated group was administered topically with $10 \%$ of TQ (TQ powder; Sigma Chemicals, USA) in petroleum jelly $(\mathrm{w} / \mathrm{v})$. TQ was administered on alternate days for 14 days.

\section{Wound contraction}

Photographs of wounds of all the rats were taken and respective wound area measurements were determined using Image $\mathrm{J} 1.48 \mathrm{v}$ software (National Institute Health, USA). The percentage of wound contractions were calculated using formula as previously described [15]: Rate of wound contraction $=[$ (original wound area-open area on final day $] /$ original wound area] $\times 100 \%$.

\section{Histological observation}

At post-wounding day 14, animals were euthanized using carbon dioxide asphyxiation, and the wound granulated tissue was harvested and fixed in 3.7\% neutral buffered formaldehyde for at least $36 \mathrm{hrs}$ for histological analysis. Tissues were then processed and embedded in paraffin wax. Serial sections of $10 \mu \mathrm{m}$ thick were cut and stained with hemotoxylin for histological investigation. Images were visualized and captured under digital microscope Olympus ix73 with the aid of cellsens software. Observation was in accordance to relative expectation of size, shape, and color of cells in hemotoxylin and eosin staining [16].

\section{Statistical analysis}

Statistical significance was determined using one-way ANOVA, followed by Tukey's HSD post hoc test. Data were represented as the mean \pm SEM, and the difference was considered significant at $\mathrm{p}<0.05$.

\section{RESULTS}

\section{Effect of blood glucose and body weight in alloxan treatment}

Alloxan-induced rats which were confirmed hyperglycemic were used in the experiment. From day 0 of experimental study, rats showed that there were no significant trends in their blood glucose levels and body weight measurements at weekly intervals (Table 1). The rats demonstrated no fluctuations in blood glucose levels and body weights during the course of the experiment.

\section{Gross observation of wound contraction}

Macroscopic evaluation of wound healing progression in Fig. 1a characterized the early formation and shedding of scabs as well as the size of the wound site through wound contraction measurement. As wound healing progress, there are noticeable differences at postwounding day 7 and 14 between the experimental groups. Evidently, $\mathrm{TQ}$ is shown to decelerate wound healing after 7 days of treatment and is further decelerated at post-wounding day 14. Although insignificant, average percentage of wound contraction in Fig. 1b supports the wound healing deceleration in TQ treatment. In addition, there is noticeable increase in wound contraction at post-wounding day 3 in effect of TQ treatment.

Histological observation of wound granulation at post-wounding day 14

Delayed wound healing model as expected in diabetes-induced rats, both groups have shown unorganized collagen depositions (Fig. 2). In addition, hematoxylin staining shows visible well-developed capillary systems in both experimental groups. Furthermore, both groups show the infiltration of inflammatory cells and fibroblasts. This indicates both are in the active proliferation phase.
However, there are noticeable morphological differences in cell infiltration levels, suggesting they are not in the same timeline phase with one another (Fig. 2). In the control group, histological assessment shows relatively low infiltration of inflammatory cells in comparison to TQ-treated group. The reduction of inflammatory cells in control group indicates wound healing is faster than the wound healing in TQtreated group. Furthermore, high infiltration of fibroblast in control group shows that wound healing is actively progressing, exiting the proliferation phase. Low infiltration of fibroblast in TQ-treated group indicates the beginning timeline of fibroblast recruitment, thus a little behind from exiting the proliferation phase.

\section{DISCUSSION}

Our study primarily focuses on the effect of TQ in treating delayed chronic wound healing as a result of metabolic impairment of diabetes. Our results have shown possible selective effect posed on different wound healing cascades. Wound healing is a dynamic and complex system; thus, repair processes are in stages but overlapping cascades [17]. During these cascade events, wound permeation differs

Table 1: Average blood glucose levels and body weights

\begin{tabular}{llllllll}
\hline \multirow{2}{*}{ Group } & \multicolumn{3}{c}{ Blood glucose (mg/dL) } & & \multicolumn{2}{c}{ Body weight (g) } \\
\cline { 2 - 4 } \cline { 6 - 8 } & Day 0 & Day 7 & Day 14 & & Day 0 & Day 7 & Day 14 \\
\hline Control & $512 \pm 45$ & $495 \pm 43$ & $539 \pm 34$ & & $207 \pm 9$ & $207 \pm 9$ & $207 \pm 9$ \\
TQ & $513 \pm 46$ & $516 \pm 50$ & $518 \pm 34$ & & $184 \pm 10$ & $184 \pm 10$ & $184 \pm 10$ \\
\hline
\end{tabular}

Data are presented as mean \pm SEM ( $n=5$ per group)

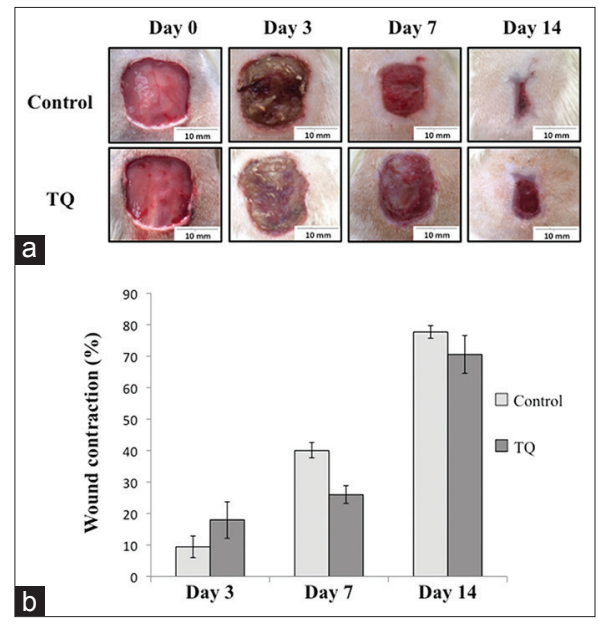

Fig. 1: (a) Wound healing progression in diabetic models with vehicle (control) and TQ treatments at given post-wounding time points, (b) average percentage of wound contractions at postwounding day 3,7 , and 14 ; data are presented as mean \pm SEM ( $n=5$ for each group)

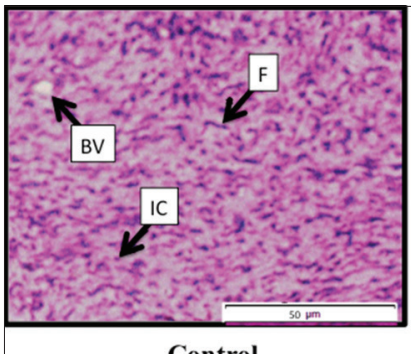

Control

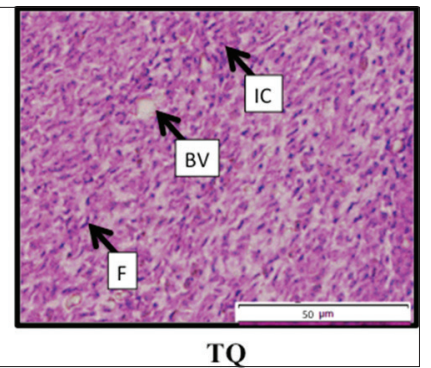

TQ
Fig. 2: Histological section of wound granulation tissues of control and TQ-treated groups on post-wounding day 14 (arrows: BV: Blood vessel; F: Fibroblast, IC: Inflammatory cell) 
greatly due to physiological changes and transition from one phase to another. For instance, the behavior of drug for vasculature uptake is higher in a vasodilated environment compare to vasoconstricted environment [18].

Our result shows that for the early wound healing cascades, TQ has been effective in accelerating wound healing. During this early phase, an initial acute inflammatory response is onset to combat harmful stimuli [19]. This usually lasted for 3 days, and is simulated as our post-wounding day 3 in wound model. This acute inflammatory response is predominantly a non-specific immune response against infection and injury [20]. Neutrophils and macrophage infiltrations are important in the acute inflammatory phase of wound healing as these cells remove debris and kill bacteria through phagocytosis [21,22]. Moreover, this cascade is important to prepare the wound bed for subsequent recruitment and proliferation of cells; however, excessive inflammatory response will impair tissue granulation processes such as angiogenesis $[17,23]$

A study has shown that dysfunctional proinflammatory cellular function in hyperglycemic microenvironment hinders suboptimal interaction of reepithelialization process [24]. This shows that hyperglycemic metabolic impairment affects wound healing ability. Chronic wound healing in diabetic patients usually manifests badly in diabetic ulcer, and this occurs as a consequence of excess reactive oxygen species (ROS) and dysfunctional inflammatory function $[21,25]$. It is usually characterized by continual influx of neutrophils that release cytotoxic enzymes and inflammatory mediators that cause damage to surrounding tissue [26]. This response leads to a progressive shift in the type of cells present in the area of inflammation; thus, simultaneously destruct and heal tissue concurrently [19]. These, in turn, produce an imbalance of ROS status and lead to oxidative stress [26].

TQ has been documented as having antioxidant and anti-inflammatory effects effective for inflammatory diseases [20]. A study of metabolic impaired rats treated with curcumin, which also possessed antioxidant and anti-inflammatory effects show the acceleration of wound healing by relieving the excessive oxidative stress and minimize cytotoxic degradative environment in diabetic wound healing model [26]. In addition, improved wound morphology was observed from a different study, indicating a prominent role of the cell infiltrations and antioxidant activity [27]. TQ is also well known for its antimicrobial effect and thus effective in minimizing local inflammatory response and infection. A study of wound healing in burn model shows that these inflammatory and antimicrobial effects are responsible for accelerated wound healing capability of TQ [12]. However, more thorough investigation is needed to determine the role of TQ during the early phase of the wound healing (post-wounding day 3 ).

Alternatively, TQ is widely used and deemed effective in combating cancer and tumor, as opposed to the direction we want to achieve, for wound healing therapy. Early prognosis indicates that the disruption of angiogenic function in cancer cell is thought to be selective, affecting only the cancer cells, but not the normal cell $[28,29]$. This predicted selective effect of TQ was based on study showing toxicity in normal cells at the minimal level [30]. This is probably due to the quiescent nature of wound vascularization [29]. However, anticancer therapy using antiangiogenic medication shows interuption in wound healing processes as its cutaneous side effects [19]

Our study demonstrated a possible cutaneous effect from TQ treatment during the active angiogenesis process in our wound healing model at post-wounding days 7 and 14. During the early granulation phase, concurrent phases display the transitioning of wound healing event from inflammatory phase to proliferative phase indicated by the reduction of inflammatory cells. Myofibroblasts and microvessels are more predominant in wound healing, with peak at 7 days and progressively reduce overtime [31]. The granulation phase started when angiogenesis begin to occur, establishing a new vascular network [29].
This organization of vascular system provides necessary oxygen and nutrient supplements for wound healing repair [22]. Angiogenesis induction in endothelial cells is mediated by angiogenic factors such vascular endothelial growth factor (VEGF) [29]. These factors induce cell migration and proliferation of endothelial cells and thus direct capillary tube morphogenesis and vascular remodeling [29].

Inadequate angiogenesis independently leads to delay wound healing, and this is not so uncommon to people with diabetes [29,32]. Hyperglycemia induces pathological changes in endothelial function resulting to abnormal angiogenesis [33]. The local metabolic environment is impaired by diabetes manifesting macro- and microvascular dysfunction that contributes to delayed wound healing [23]. The inhibition of endothelial response to injury may manifest occurrence of wound dehiscence, bleeding, and wound infection [29]. It is worth noting that impaired wound healing in diabetes is not only just characterized by a decrease in angiogenic function but also in reduction of endothelial progenitor cell recruitment [5]. Saito et al. had discovered that the subsets of endothelial progenitors are altered in diabetic rats, resulting in impaired endothelial vascular differentiation [34].

As angiogenesis function is disrupted, wound healing becomes chronically inflamed. Chronic inflammation hinders progression to subsequent granulation phase due to imbalance ROS and oxidative stress in wound [21]. This causes lipid peroxidation of organelles and cellular membranes, responsible to disrupt the function of fibroblast and endothelial cells [35]. In addition, lipid peroxidation is suspected to impair VEGF expression for normal angiogenesis process [35]. Antioxidant agents are effective to combat excessive ROS activity and thus minimize lipid peroxidation $[22,25]$. TQ has shown to improve endothelial function by reducing oxidative stress [36]. However, its antiangiogenic effect is probably stronger in decelerating wound healing.

To the best of our knowledge, the pathway of TQ in decelerating wound healing progression still remains unknown. Nevertheless, our histopathological analysis on post-wounding day 14 has indicated that wound healing is decelerated in TQ-treated group. However, more follow-up study is needed, and we suggest the utilization of oxidative stress and vascular motif to potentiate better investigation series.

\section{CONCLUSION}

Our data show that TQ accelerate wound healing in diabetic model during the inflammatory phase, this is possibly due to its well-known antioxidant, anti-inflammatory, and antimicrobial properties. However, TQ shifts its function, as wound healing is severely decelerated during granulation phase. We speculate the deceleration of wound healing is attributed due to TQ's antiangiogenic property.

\section{ACKNOWLEDGMENT}

The study was supported by Universiti Brunei Darussalam.

\section{REFERENCES}

1. Günter CI, Machens HG. New strategies in clinical care of skin wound healing. Eur Surg Res 2012;49:16-23.

2. Eming SA. Etiology and pathophysiology. Ther Skin Dis 2010;735-51. DOI: 10.1007/978-3-540-78814-0 62.

3. Kolluru GK, Bir SC, Kevil CG. Endothelial dysfunction and diabetes: Effects on angiogenesis, vascular remodeling, and wound healing. Int J Vasc Med 2012;2012:918267.

4. Bowling FL, Rashid ST, Boulton AJ. Preventing and treating foot complications associated with diabetes mellitus. Nat Rev Endocrinol 2015;11:606-16.

5. Catrina SB, Zheng X. Disturbed hypoxic responses as a pathogenic mechanism of diabetic foot ulcers. Diabetes Metab Res Rev 2016;32 Suppl 1:179-85.

6. Casella S, Bielli A, Mauriello A, Orlandi A. Molecular pathways regulating macrovascular pathology and vascular smooth muscle cells phenotype in Type 2 diabetes. Int J Mol Sci 2015;16:24353-68. 
7. Amin MA, Abdel-Raheem IT. Accelerated wound healing and antiinflammatory effects of physically cross linked polyvinyl alcoholchitosan hydrogel containing honey bee venom in diabetic rats. Arch Pharm Res 2014;37:1016-31.

8. Banu A, Noorul Hassan MM, Rajkumar J, Srinivasa S. Spectrum of bacteria associated with diabetic foot ulcer and biofilm formation: A prospective study. Australas Med J 2015;8:280-5.

9. Cordell GA. Phytochemistry and traditional medicine - The revolution continues. Phytochem Lett. DOI: 10.1016/j.phytol.2014.06.002.

10. El-Dakhakhny M. Studies on the chemical constitution of Egyption N. sativa L. Seeds. Planta Med 1963;11(2):465-70.

11. Ab Rahman MR, Abdul Razak F, Mohd Bakri M. Evaluation of wound closure activity of Nigella sativa, Melastoma malabathricum, Pluchea indica, and Piper sarmentosum extracts on scratched monolayer of human gingival fibroblasts. Evid Based Complement Alternat Med 2014;2014:190342.

12. Selçuk CT, Durgun M, Tekin R, Yolbas L, Bozkurt M, Akçay C, et al. Evaluation of the effect of thymoquinone treatment on wound healing in a rat burn model. J Burn Care Res 2013;34:e274-81.

13. McLaughlin PJ, Pothering CA, Immonen JA, Zagon IS. Topical treatment with the opioid antagonist naltrexone facilitates closure of full-thickness wounds in diabetic rats. Exp Biol Med (Maywood) 2011;236:1122-32.

14. Umachigi SP, Jayaveera KN, Kumar CK, Kumar GS, Swamy BM, Kumar DV. Studies on wound healing properties of Quercus infectoria. Trop J Pharm Res 2008;7(1):913-9.

15. Shi HX, Lin C, Lin BB, Wang ZG, Zhang HY, Wu FZ, et al. The anti-scar effects of basic fibroblast growth factor on the wound repair in vitro and in vivo. PLoS One 2013;8:e59966.

16. Eroschenko VP. DiFiore's Atlas of Histology with Functional Correlations. USA: Lippincott Williams \& Wilkins; 2008.

17. Brown DA, Gibran NS. History of wound care. In: Jeschke MG, Kamolz L, Sjöberg F, Wolf SE, editors. Handbook of Burns Volume 1: Acute Burn Care. Vienna: Springer Vienna; 2012. p. 325-8

18. Jepps OG, Dancik Y, Anissimov YG, Roberts MS. Modeling the human skin barrier--towards a better understanding of dermal absorption. Adv Drug Deliv Rev 2013;65(2):152-68.

19. Hadagali MD. Chua LS. The anti-inflammatory and wound healing properties of honey. Eur Food Res Technol 2015;239(6):1003-14.

20. Woo CC, Kumar AP, Sethi G, Tan KH. Thymoquinone: Potential cure for inflammatory disorders and cancer. Biochem Pharmacol 2012:83:443-51.

21. Kasuya A, Tokura Y. Attempts to accelerate wound healing. J Dermatol Sci 2014;76:169-72

22. Valizadeh R, Hemmati AA, Houshmand G, Bayat S, Bahadoram M. Wound healing potential of Althaea officinalis flower mucilage in rabbit full thickness wounds. Asian Pac J Trop Biomed 2015;5(11):937-43.

23. Henshaw FR, Boughton P, Lo L, McLennan SV, Twigg SM. Topically applied connective tissue growth factor/CCN2 improves diabetic preclinical cutaneous wound healing: Potential role for CTGF in human diabetic foot ulcer healing. J Diabetes Res 2015;2015:236238.

24. Huang SM, Wu CS, Chao D, Wu CH, Li CC, Chen GS, et al. Highglucose-cultivated peripheral blood mononuclear cells impaired keratinocyte function via reduced IL-22 expression: Implications on impaired diabetic wound healing. Exp Dermatol 2015;24:639-41.

25. Zhang Z, Cao G, Sha L, Wang D, Liu M. The efficacy of sodium aescinate on cutaneous wound healing in diabetic rats. Inflammation 2015;38:1942-8.

26. Kant V, Gopal A, Pathak NN, Kumar P, Tandan SK, Kumar D. Antioxidant and anti-inflammatory potential of curcumin accelerated the cutaneous wound healing in streptozotocin-induced diabetic rats. Int Immunopharmacol 2014;20:322-30.

27. Farahpour M, Emami P, Ghayour S. In vitro antioxidant properties and wound healing activity of hydroethanolic turmeric rhizome extract (Zingiberaceae). Int J Pharm Pharm Sci 2014;6(8);474-8.

28. Alenzi FQ, Altamimi MA, Kujan O, Tarakji B, Tamimi W, Bagader O, et al. Antioxidant properties of Nigella sativa. J Mol Genet Med 2013;7(3):1000077.

29. Bodnar RJ. Anti-angiogenic drugs: Involvement in cutaneous side effects and wound-healing complication. Adv Wound Care (New Rochelle) 2014;3:635-46.

30. Gali-Muhtasib H, Roessner A, Schneider-Stock R. Thymoquinone: A promising anti-cancer drug from natural sources. Int J Biochem Cell Biol 2006;38:1249-53.

31. Almeida BM, Nascimento MF, Pereira-Filho RN, Melo GC, Santos JC, Oliveira CR, et al. Immunohistochemical profile of stromal constituents and lymphoid cells over the course of wound healing in murine model. Acta Cir Bras 2014;29:596-602.

32. Hirapara H, Ghori V, Anovadiya A, Tripathi C. Effect of amlodipine and enalpril on wound healing in diabetic wistar albino rats. Int J Pharm Pharm Sci 2016;8(7);257-61.

33. Bagdas D, Etoz BC, Gul Z, Ziyanok S, Inan S, Turacozen O, et al. In vivo systemic chlorogenic acid therapy under diabetic conditions: Wound healing effects and cytotoxicity/genotoxicity profile. Food Chem Toxicol 2015;81:54-61.

34. Saito H, Yamamoto Y, Yamamoto H. Diabetes alters subsets of endothelial progenitor cells that reside in blood, bone marrow, and spleen. Am J Physiol Cell Physiol 2012;302:C892-901.

35. Moghadamtousi SZ, Rouhollahi E, Hajrezaie M, Karimian H, Abdulla MA, Kadir HA. Annona muricata leaves accelerate wound healing in rats via involvement of Hsp70 and antioxidant defence. Int J Surg 2015;18:110-7.

36. Shabana A, El-Menyar A, Asim M, Al-Azzeh H, Al Thani H. Cardiovascular benefits of black cumin (Nigella sativa). Cardiovasc Toxicol 2013;13(1):9-21. 